\title{
Haploid yeast cells undergo a reversible phenotypic switch associated with chromosome II copy number
}

Polina Drozdova ${ }^{1 *}$, Ludmila Mironova ${ }^{1}$ and Galina Zhouravleva ${ }^{1,2}$

From The International Conference on Bioinformatics of Genome Regulation and StructurelSystems Biology (BGRSISB-2016)

Novosibirsk, Russia. 29 August-2 September 2016

\begin{abstract}
Background: SUP35 and SUP45 are essential genes encoding polypeptide chain release factors. However, mutants for these genes may be viable but display pleiotropic phenotypes which include, but are not limited to, nonsense suppressor phenotype due to translation termination defect. $\left[\mathrm{PS} \mathrm{I}^{+}\right]$prion formation is another Sup35p-associated mechanism leading to nonsense suppression through decreased availability of functional Sup35p. $\left[\mathrm{PSI}^{+}\right]$differs from genuine sup35 mutations by the possibility of its elimination and subsequent re-induction. Some suppressor sup35 mutants had also been shown to undergo a reversible phenotypic switch in the opposite direction. This reversible switching had been attributed to a prion termed $\left[/ S P^{+}\right]$. However, even though many phenotypic and molecular level features of $\left[/ S P^{+}\right]$were revealed, the mechanism behind this phenomenon has not been clearly explained and might be more complex than suggested initially.
\end{abstract}

Results: Here we took a genomic approach to look into the molecular basis of the difference between the suppressor (Isp-) and non-suppressor $\left(\mathrm{Isp}^{+}\right)$phenotypes. We report that the reason for the difference between the $\mathrm{Isp}^{+}$and the Isp ${ }^{-}$phenotypes is chromosome II copy number changes and support our finding with showing that these changes are indeed reversible by reproducing the phenotypic switch and tracking karyotypic changes. Finally, we suggest mechanisms that mediate elevation in nonsense suppression efficiency upon amplification of chromosome II and facilitate switching between these states.

Conclusions: (i) In our experimental system, amplification of chromosome II confers nonsense suppressor phenotype and guanidine hydrochloride resistance at the cost of overall decreased viability in rich medium. (ii) SFP1 might represent a novel regulator of chromosome stability, as SFP1 overexpression elevates frequency of the additional chromosome loss in our system. (iii) Prolonged treatment with guanidine hydrochloride leads to selection of resistant isolates, some of which are disomic for chromosome II.

Keywords: Aneuploidy, Translation termination, Nonsense suppression, SUP35, Yeast, Chromosome instability

\footnotetext{
* Correspondence: p.drozdova@spbu.ru

${ }^{1}$ Department of Genetics and Biotechnology, St. Petersburg State University,

199034, Universitetskaya nab. 7-9, St. Petersburg, Russia

Full list of author information is available at the end of the article
} 


\section{Background}

Translation is a very important and energy-demanding process for all living cells including the yeast Saccharomyces cerevisiae. Synthesis of ribosomal components is ultimately the main activity of the cell. Apart from copious rRNA molecules produced by RNA polymerases I and III, mRNAs encoding ribosomal proteins and ribosome assembly factors comprise at least $60 \%$ of the transcripts produced by RNA polymerase II [1, 2]; in addition, not only ribosome components but many other proteins are required for efficient protein synthesis. No wonder that this system is tightly regulated, and many of its components play a role in this regulation [3].

The process of translation ceases when the translating ribosome encounters one of three stop codons. This step, termed termination, is ensured by the release factors Sup35p (eRF3) and Sup45p (eRF1). These proteins form a complex resembling tRNA; they act by binding to the stop codon and prompting release of the newly synthesized protein to the cytoplasm. Binding of tRNAs and release factor complex to the stop codons exists in a dynamic equilibrium which may shift if levels of any of these components are altered. If the equilibrium shifts in a cell bearing a gene with a premature termination codon (nonsense mutation), the effect of this mutation may be partially compensated for. This phenomenon is referred to as nonsense suppression. The most obvious reasons for nonsense suppression are mutations in tRNA and release factor genes [4]; in addition, partial inactivation of Sup35p deposited in amyloid aggregates $\left(\left[\mathrm{PSI}^{+}\right],[5]\right)$ was shown to result in a similar phenotype. Mutations in the release factor genes may be adaptive or counter-adaptive depending on the growth conditions, as easily illustrated by a simple example of a sup35 mutation partially restoring growth of an ade1-14 mutant strain on adenine dropout media but leading to sensitivity of this strain to elevated temperatures.

Rich collections of spontaneous suppressor mutations in the SUP35 and SUP45 genes have been obtained and extensively characterized in several strain backgrounds with different suppressible nonsense mutations [6-9], one of them being 2V-P3982 with ade1-14 (UGA), his7-1 (UAA) and lys2-87 (UGA) and non-suppressor Ade $^{-}$His $^{-}$Lys $^{-}$phenotype. Two sup35 strains from this collection were shown to switch from suppressor phenotype $\left(\mathrm{Ade}^{+} \mathrm{His}^{+} \mathrm{Lys}^{+}\right.$) to non-suppressor $\left(\mathrm{Ade}^{ \pm} \mathrm{His}^{-} \mathrm{Lys}^{-}\right.$) phenotype spontaneously. Curiously, the non-suppressor phenotype was characterized by non-Mendelian inheritance, could be eliminated on media containing guanidine hydrochloride $(\mathrm{GuHCl})$ and re-appeared after $\mathrm{GuHCl}$ caused elimination, similar to known prions [10].

This prion-like determinant associated with reduced nonsense suppression efficiency was designated $\left[I S P^{+}\right]$for "inversion of suppressor phenotype". Despite being similar to a prion with a clear link to a transcriptional regulator Sfp1p, a potentially prionogenic protein enriched in asparagine and glutamine residues $[11],\left[I S P^{+}\right]$has a number of features which distinguish it from most "canonical" prions. Its propagation does not depend on the Hsp104 chaperone [10], which is required for propagation of other prions [12], and deletion of the SFP1 gene conveys a phenotype drastically different from the $\left[I S P^{+}\right]$phenotype [11]. In addition, $\left[I S P^{+}\right]$strains have been found to contain not only the sup35-25 suppressor mutation, but also a missense substitution sup45-400. The combination of sup35 and sup 45 mutations contributes to the development the suppressor phenotype, since introduction of plasmid-borne wild-type SUP45 into the $\left[I S P^{+}\right]$strain leads to $\left[i s p^{-}\right]$-like suppressor phenotype [13].

In presence of pre-existing suppressors, translation termination efficiency may be modulated by multiple factors including the $\Psi$ factor $[14]$, i.e. the $\left[P S I^{+}\right]$prion [15], or an additional chromosome [16]. Similar to suppressor mutations and prions, presence of an additional chromosome may confer adaptiveness or counteradaptiveness depending on the conditions tested [17, 18]. Many natural isolates were shown to be aneuploid [19-21], and in some natural isolates prions were revealed [22, 23], which probably reflects the utility of these traits.

In this work, we show that in an unstable strain copy number of chromosome II can modulate nonsense suppressor phenotype and resistance to guanidinium chloride. Our results also suggest that Sfp1p, a transcriptional regulator, is implicated in maintenance of chromosome stability.

\section{Results}

Isp $^{+}$and Isp $^{-}$isolates used for transcriptional profiling differ in copy number of chromosomes II and IX, and genome sequencing confirms this result

$\left[\mathrm{ISP}^{+}\right]$has been studied in a group of closely related strains mostly ascending to 25-2V-P3982 (a 2V-P3982 derivative bearing the sup35-25 mutation [10]). In recent works, the 25-25-2V-P3982 strain was used, which had been derived from 25-2V-P3982 through mating type switch [13]. The strain has been stored in glycerol stocks with several episodes of partial defreezing; so, genetic identity of the original strains of the early 2000s and the strains used in this work cannot be guaranteed. For this reason, throughout this work we will refer to sup35-25 isolates as either $\mathrm{Isp}^{+}$or $\mathrm{Isp}^{-}$. $\mathrm{Isp}^{+}$stands for $\mathrm{His}^{-} \mathrm{Lys}^{-}$ phenotype while $\mathrm{Isp}^{-}$refers to $\mathrm{His}^{+} \mathrm{Lys}^{+}$phenotype; isolates of both types are $\mathrm{Ade}^{+}$.

Recently, we compared the transcriptional profiles of two isolates presumed to be $\left[I S P^{+}\right]$and $\left[i s p^{-}\right]$[24]. In this work, we will refer to these isolates as p2 (Isp ${ }^{+}, \mathrm{p}$ for 'plus') and $\mathrm{m} 2$ ( $\mathrm{Isp}^{-}, \mathrm{m}$ for 'minus'), respectively (see below). We found a small number $(\sim 300)$ of differentially 
expressed genes which fall into two classes, those upregulated in the $\mathrm{Isp}^{+}$isolate and those upregulated in the Isp $^{-}$one. While genes of the first group formed particular functional clusters associated with nutrient assimilation and metal ion import, the second group of genes was not enriched in any particular functional groups and thus similarly lacked common reason for changing expression [24]. However, genes of the second group were united by another feature, their chromosomal location. Hypergeometric test showed they were significantly enriched in genes located on chromosomes II and IX ( $p=3 \times 10^{-118}$ and $p=2 \times 10^{-47}$, respectively). Plotting relative $\left(\mathrm{Isp}^{-} / \mathrm{Isp}^{+}\right)$gene expression values against gene position in the reference genome clearly illustrated the same tendency (Fig. 1a).

In order to check how these data correspond to the previously reported gene expression data for disomic strains, we exploited the vast body of evidence generated for twelve different haploid disomic strains and accumulated in the work of Dephoure et al. [25]. Indeed, the distribution of gene expression values looked very similar (Additional file 1: Figure S1), even though overall Pearson correlation of the expression profile of the Isp ${ }^{-}$isolate with expression profiles of strains disomic for only chromosome II or only chromosome IX [25] was not high ( $r=0.38$ and $r=0.20$, respectively).

However, sequencing read depth could provide an additional (and possibly more reliable) measure of chromosome copy number than the mRNA level. It could also indicate whether the isolates (even though unlikely) differ in some point mutations which contribute to the phenotype. Two isolates were chosen for whole genome sequencing. The Isp ${ }^{-}$isolate was a copy of same Isp $^{-}$isolate used for transcription profile analysis ( $\mathrm{m} 2)$, which had been passaged on YEPD and then stored at $-80{ }^{\circ} \mathrm{C}$ as a glycerol stock. The Isp ${ }^{+}$isolate (p3) was a derivative of $\mathrm{m} 2$ obtained with transient SFP1 overexpression (see Fig. 2). First, depth of coverage analysis was performed (Fig. 1b, c). We indeed found a difference in chromosome II and IX copy number. Surprisingly, the $\mathrm{Isp}^{+}$isolate (p3) showed non-integer coverage for chromosome IX (Fig. 1c), which probably means that it consisted of a mixture of monosomic and disomic cells. In agreement to the gene expression values,
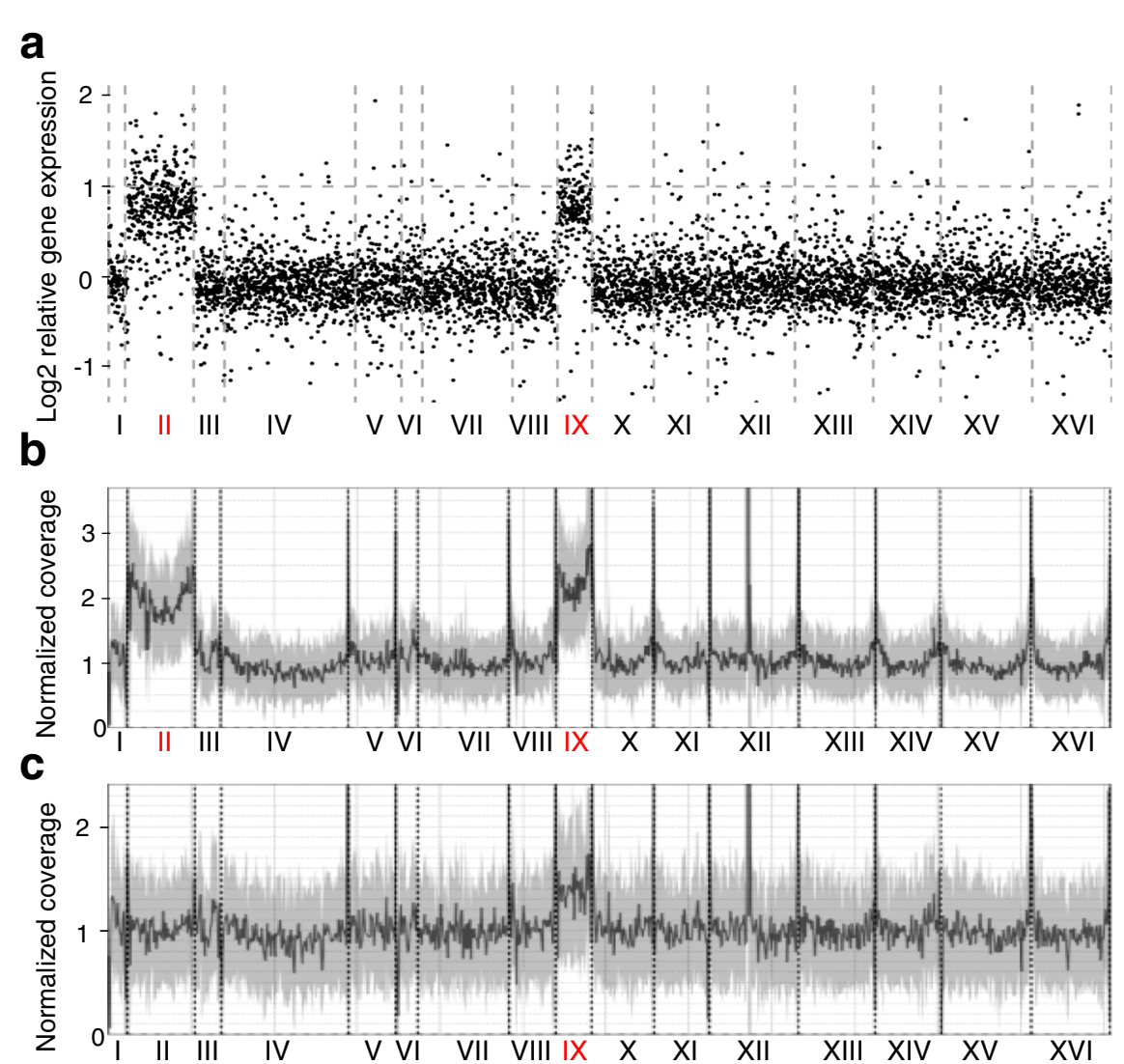

Fig. $\mathbf{I} \mathrm{Isp}^{+}$and $\mathrm{Isp}^{-}$isolates differ in copy number of chromosomes II and IX. a Expression values for all the genes in an Isp ${ }^{-}$isolate (m2) relative to an $\mathrm{Isp}^{+}$one (p2), sorted by chromosome. $\mathbf{b}$ Normalized coverage throughout the reference genome for an Isp ${ }^{-}$isolate (m2). $\mathbf{c}$ Normalized coverage throughout the reference genome for an $\mathrm{Isp}^{+}$isolate (p3). Chromosome numbers are indicated at the bottom. Full datasets are available in Additional file 2: Table S1 


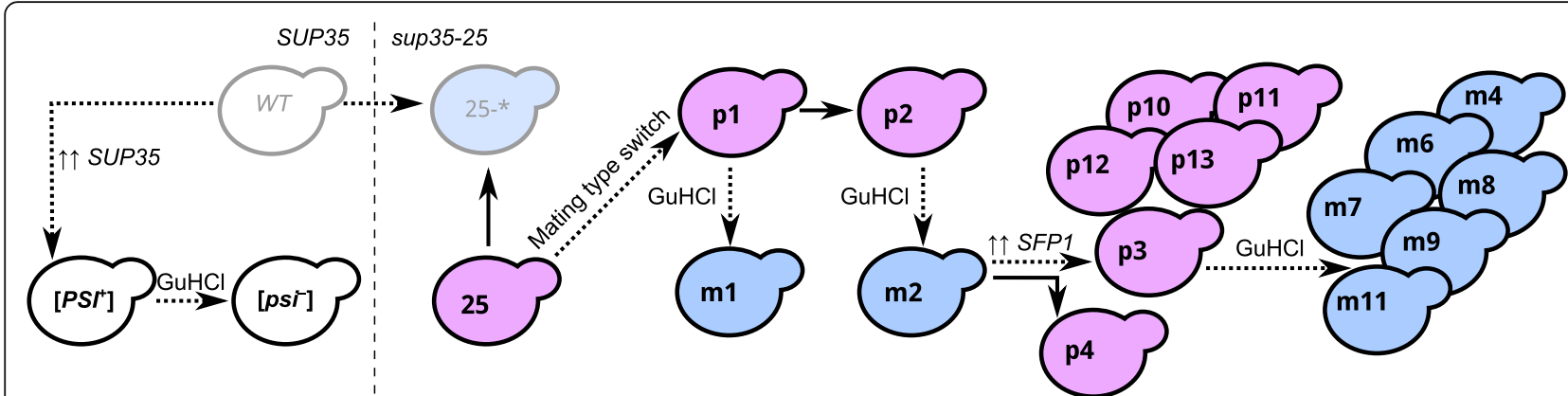

Fig. 2 Relationship of the isolates used in this work. Grey contour, clones no longer existing in the collection; black contour, isolates analyzed in our work. The dotted line separates SUP35 SUP45 and sup35-25 sup45-400 clones. Blue background signifies Lys ${ }^{+}$or Lys $^{+} H_{i s}{ }^{+}$ phenotype (Isp ${ }^{-}, \mathrm{m}$ for 'minus'); purple background signifies $\mathrm{Lys}^{-} \mathrm{His}^{-}$(Isp ${ }^{+}, \mathrm{p}$ for 'plus') phenotype. GuHCl, guanidine hydrochloride treatment. Solid arrows signify passaging without treatment; dotted arrows signify GuHCl treatment or transformation (indicated above). $\uparrow \uparrow$ SFP1, SFP1 overexpression with subsequent plasmid loss for isolation of Isp ${ }^{+}$clones. $\uparrow \uparrow$ SUP35, SUP35 overexpression with subsequent plasmid loss for $\left[\mathrm{PSI}^{+}\right]$induction

the Isp $^{-}$isolate $(\mathrm{m} 2)$ showed approximately two-fold higher coverage for chromosomes II and IX than for the other chromosomes (Fig. 1b). In addition, we compared single nucleotide variation data for the two isolates and did not find any "suspicious" positions which could have been selected for; chromosome II of the $\mathrm{m} 2$ genome was homozygous for the sup45-400, his7-1 and lys2-87 alleles.

\section{Transitions between Isp ${ }^{+}$and Isp ${ }^{-}$phenotypes strongly correlate with changes in chromosome II number}

As the molecular difference between $\mathrm{Isp}^{+}$and $\mathrm{Isp}^{-}$isolates turned out to be distinct from the prion-like determinant described previously, we asked whether it was also reversible. Thus, it was crucial to establish whether the chromosome copy number difference would be reproduced in newly obtained $\mathrm{Isp}^{+}$and $\mathrm{Isp}^{-}$isolates.

There are two known means to obtain $\mathrm{Isp}^{+}$clones from Isp $^{-}$ones, namely spontaneous appearance happening at a frequency of about 1 per 10,000 cells [10] and SFP1 overexpression elevating this frequency $\sim 700$-fold [11]. There is only one known way to obtain Isp ${ }^{-}$isolates from $\mathrm{Isp}^{+}$ ones, $\mathrm{GuHCl}$ treatment [10]. We employed these three methods and used $\mathrm{m} 2$ to obtain one spontaneous $\mathrm{Isp}^{+}$isolate (p4; Fig. 2, lower part) and four more $\mathrm{Isp}^{+}$isolates after transient overexpression of SFP1 (isolates p10, p11, p12, p13; Fig. 2, lower part); p3 was passaged on GuHClcontaining media five times to select six Isp ${ }^{-}$isolates $(\mathrm{m} 4$, $\mathrm{m} 5, \mathrm{~m} 7, \mathrm{~m} 8, \mathrm{~m} 9$ and $\mathrm{m} 11$; Fig. 2, lower part). In addition, we used the oldest glycerol stocks available to recover Isp ${ }^{+}$ and Isp $^{-}$isolates $\mathrm{p} 1$ and $\mathrm{m} 1$. Finally, we tried to obtain strains parental to 25-25-2V-P3982 (sup35-25 MATa) and

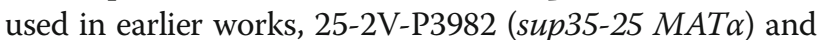

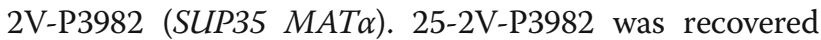
from glycerol stock (Fig. 2, isolate 25). No stocks of the original 2V-P3982 strain were available but its $\left[\mathrm{PSI}^{+}\right]$derivative was stored; we recovered it (Fig. 2, isolate $\left[P S I^{+}\right]$) and also treated it with $\mathrm{GuHCl}$ to obtain a prionless strain (Fig. 2, isolate $\left[p s i^{-}\right]$).

Then, we employed microarray-based comparative genomic hybridization $(\mathrm{aCGH})$ to infer copy number of each chromosome in each of these isolates (Table 1). Chromosome II copy number perfectly correlates with the $\mathrm{Lys}^{+} / \mathrm{Lys}^{-}$phenotype of the particular isolate: to sum up, all $7 \mathrm{Isp}^{+}$isolates tested were monosomic for chromosome II while all $8 \mathrm{Isp}^{-}$isolates had this chromosome amplified. In addition, we found some variability in copy number of chromosomes I, IX and XIV (Table 1). We could not associate chromosome I or XIV disomy with any changes in nonsense suppression efficiency. Chromosome IX copy number might influence his7-1 suppression efficiency since $\mathrm{m} 1$, the only $\mathrm{Isp}^{-}$isolate monosomic for chromosome IX in our analysis, is characterized by poorer growth on histidine dropout medium than the other Isp

Table 1 Isp ${ }^{-}$isolates differ from Isp ${ }^{+}$ones by chromosome II copy number

\begin{tabular}{|c|c|c|}
\hline Isolate name & Phenotype & Karyotype \\
\hline$\left[P S I^{+}\right]$ & Ade $^{+} \mathrm{His}^{-} \mathrm{Lys}^{-}$ & euploid \\
\hline$[p s i$ & $\mathrm{Ade}^{-} \mathrm{His}^{-} \mathrm{Lys}^{-}$ & +1 \\
\hline 25, p1, p10, p11, p12, p13 & $\mathrm{Isp}^{+}$ & euploid \\
\hline p3 & & $+\mathrm{XIV}$ \\
\hline p4 & & $+I X$ \\
\hline $\mathrm{m} 1$ & $\mathrm{Isp}^{-}$ & + II \\
\hline $\mathrm{m} 2, \mathrm{~m} 4, \mathrm{m6}, \mathrm{m7}, \mathrm{m8}, \mathrm{m} 9, \mathrm{~m} 11$ & & +II, IX \\
\hline
\end{tabular}

Shown are summarized results of the aGCH analysis. For isolate names, see Fig. 2. Isp ${ }^{-}$isolates are indicated in bold. For full data, see Additional file 3: Figure S2 and Additional file 4: Table S2 
isolates; however, we consider this unlikely as the original Isp $^{-}$isolate has been previously shown to suppress both his7-1 and lys2-87 [10].

\section{Possible mechanisms for transitions between the $\mathrm{Isp}^{+}$and} Isp $^{-}$states

Our results show that Isp ${ }^{+}$clones isolated after transient SFP1 overexpression lose extra chromosomes. Thus, SFP1 overexpression might elevate the frequency of extra chromosome loss. The simplest explanation would be that SFP1 overexpression, which is toxic for the cell, might be even more toxic for disomic cells, allowing for selection of euploids. However, this was not true; moreover, SFP1 overexpression was even more toxic for Isp ${ }^{+}$ isolates (Fig. 3).

In the case of the reverse transition, $\mathrm{Isp}^{+}$to $\mathrm{Isp}^{-}$, selection for resistance to $\mathrm{GuHCl}$ may take place. [isp -] isolates had been shown to be more resistant to $5 \mathrm{mM} \mathrm{GuHCl}$ than $\left[I S P^{+}\right]$ones [10]; we confirmed this results (compare upper and lower lines at Fig. 4). Curing of prions with $\mathrm{GuHCl}$ depends on $\mathrm{Hsp} 104$ [26] but the $\left[I S P^{+}\right]$prion had been shown to be independent of Hsp104 [10]. What happened when strains were treated with $\mathrm{GuHCl}$ is unclear. We passaged an $\mathrm{Isp}^{+}$isolate on either YEPD or YEPD with $\mathrm{GuHCl}$ five times and then compared the phenotype (Fig. 4). $\mathrm{GuHCl}$ treatment led to selection for GuHCl-resistant isolates in $\mathrm{Isp}^{+}$with simultaneous selection for $\mathrm{Lys}^{+}$ $\left(\right.$ Isp $\left.^{-}\right)$clones. Thus, amplification of chromosome II might be one of the mechanisms of adjustment to $\mathrm{GuHCl}$.

\section{Mechanisms for nonsense suppression associated with chromosome II disomy}

As we show, chromosome II ploidy state correlates with the efficiency of suppressor phenotype, i.e., growth on histidine or lysine dropout media. This phenotype might be associated with marker nonsense mutations his7-1 and lys2-87 used to assess nonsense suppressor efficiency, as both genes are located on chromosome II. We chose one of these marker genes, his7-1, and checked whether introduction of an additional copy of this allele on a centromeric plasmid would produce a result similar to chromosome amplification. Indeed, an additional copy of his7-1 did elevate growth on histidine dropout medium without affecting growth on lysine dropout medium (20 independent transformants were checked; a representative clone is shown at Fig. 5). However, this effect was not strong enough to mimic the Isp ${ }^{-}$phenotype (Fig. 5, compare lines 2 and 3). Thus, $\mathrm{His}^{+}$phenotype of the Isp ${ }^{-}$isolates depends on the his7-1 copy number, but there might be other contributing factors.

\section{Discussion}

In this work we show that the phenotypic difference between the $\mathrm{Isp}^{+}$and $\mathrm{Isp}^{-}$isolates depends on chromosome II copy number. This finding is unexpected since these isolates were originally described as different in their $\left[I S P^{+}\right]$prion state $[10,11]$. The original suppressor sup35-25 isolate was not preserved, so we cannot directly determine whether it was disomic or displayed marked nonsense suppression for another reason. However, progeny of a hybrid of sup35-25 [isp $\left.{ }^{-}\right]$and SUP35 strains had shown monogenic segregation of the suppressor phenotype $[6,10]$ while a hybrid of sup35-25 [ISP $\left.{ }^{+}\right]$and SUP35 strains showed deviation from 2:2 segregation [10]. If this Isp ${ }^{-}$strain were disomic for chromosome II and the disomy contributed to the suppressor phenotype, as we see in the strain studied in this work, deviation from monogenic segregation would have been observed in the former, but not in the latter case. These data suggest that the $\mathrm{Isp}^{+} / \mathrm{Isp}^{-}$phenotypes studied in this work represent phenocopies of the original phenotypes determined by presence or absence of the $\left[I S P^{+}\right]$prion.

We have shown that transitions from $\mathrm{Isp}^{+}$to $\mathrm{Isp}^{-}$ phenotype and vice versa are indeed associated with changes in chromosome II copy number (Fig. 2 and Table 1). However, the question why these transitions happen still remains. Spontaneous appearance of $\mathrm{Isp}^{+}$ clones can be attributed to accidental loss of the extra chromosome in cell divisions facilitated by the fact that $\mathrm{Isp}^{+}$clones grow slightly faster than $\mathrm{Isp}^{-}$ones [10]. However, it is unclear why overexpression of SFP1

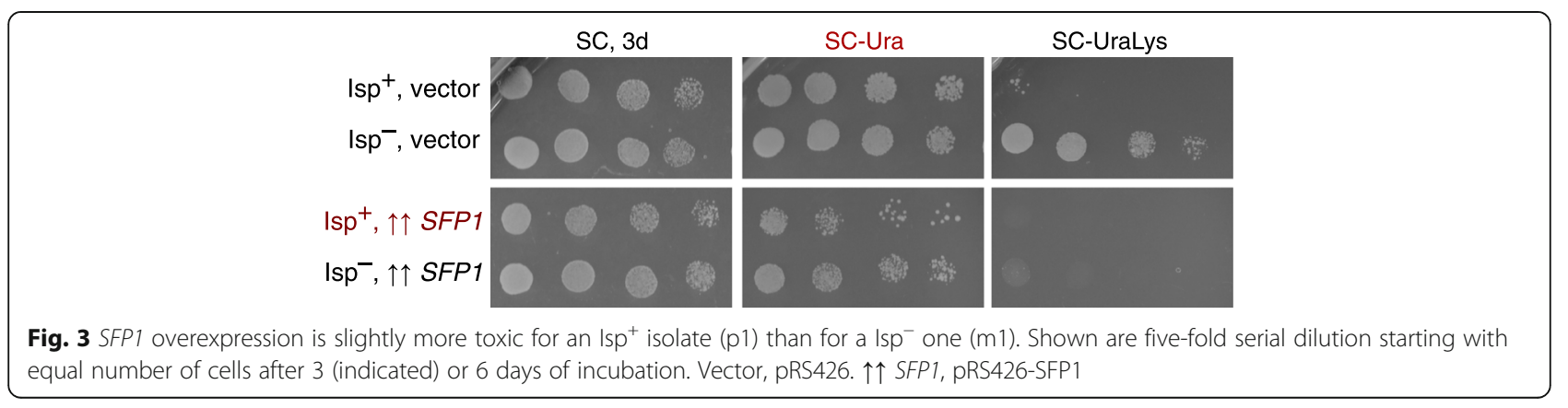




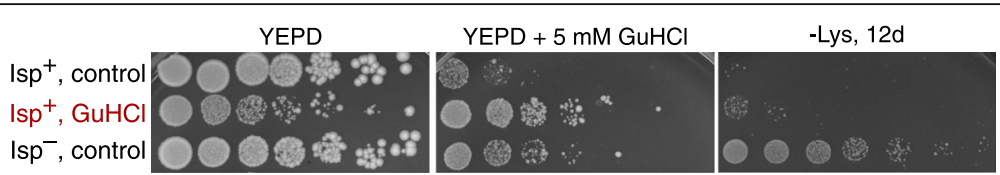

Fig. 4 The Lys ${ }^{+}$phenotype is co-selected with resistance to GuHCl. An Isp $(\mathrm{p} 1$ ) isolate was passaged on either YEPD (upper line) or YEPD with $5 \mathrm{mM} \mathrm{GuHCl}$ (middle line) five times; an Isp ${ }^{-}$isolate $(\mathrm{m} 1)$ passaged on YEPD (lower line) is shown for comparison. Shown are five-fold serial dilutions starting with equal number of cells after 6 or 12 (indicated) days of incubation

elevates the frequency of extra chromosome loss. Increased level of Sfp1p might modulate expression of some target gene regulating chromosome maintenance or Sfp1p itself might interact with such regulator. Chromosome loss can happen due to unrepaired double strand breaks or defects in chromosome segregation. SFP1 overexpression affects cell cycle, most probably causing cells to pause in G2 [27], so we can speculate that cells overexpressing SFP1 could either have more double strand breaks or be defective for double strand break repair or spindle assembly.

The reverse transition, from $\mathrm{Isp}^{+}$to Isp ${ }^{-}$, might be associated with selection for $\mathrm{GuHCl}$ resistance. It is worth emphasizing that while $5 \mathrm{mM} \mathrm{GuHCl}$ inhibits growth of yeast cells (Fig. 4, compare left and middle panels), growth of Isp $^{+}$isolates is inhibited by $5 \mathrm{mM} \mathrm{GuHCl}$ more than the growth of Isp ${ }^{-}$isolates [10], and we confirm this result (Fig. 4). $\mathrm{GuHCl}$ resistance might be caused by increased level of Hal3p (Sis2p) reported earlier [28] or also by some gene located on chromosome II. Emergence of aneuploid clones in response to a stressful condition is similar to other reported cases such as chromosome III amplification in response to heat stress or chromosome $\mathrm{V}$ amplification as an adaptation to high $\mathrm{pH}$ [17] as well as chromosome XIII disomy making gal7 strains galactose tolerant [29]. Interestingly, chromosome II disomy has been already described in some laboratory strains as a compensatory mechanism. It was shown to arise in response to polyQ toxicity due to the Sup 45 protein [30] or to provide viability for strains deleted for the hta1-htb1 locus due to the increasing dosage of the HTA2 and HTB2 genes [31]. As only some of the clones growing well on GuHCl-containing media are $\mathrm{Lys}^{+}$and further prove to be disomic for chromosome II, we suggest that there are multiple ways to adjust to high $\mathrm{GuHCl}$ concentrations toxic for the cell, one of them being chromosome II disomy associated with the Isp ${ }^{-}$phenotype.
We show that chromosome II copy number (monosomy/ disomy) perfectly correlates with the $\mathrm{Isp}^{+} / \mathrm{Isp}^{-}$phenotype of the particular isolate, suggesting that it is the reason for the difference in growth on histidine or lysine dropout media. The simplest explanation of the mechanism would be increased dosage of the his7-1 or lys2-87 mRNA, respectively. We checked this hypothesis and showed that introduction of an additional copy of his7-1 indeed leads to $\mathrm{His}^{+}$phenotype of an $\mathrm{Isp}^{+}$isolate. We can speculate that the same mechanism takes place in the case of lys2-87. However, as an Isp ${ }^{+}$isolate with two copies of his7-1 still produces slightly less $\mathrm{His}^{+}$clones than an $\mathrm{Isp}^{-}$isolate (Fig. 5); this might be caused either by the missense mutation in the plasmid-borne his7-1 copy or by influence of some other gene(s) located on chromosome II or regulated by chromosome II genes. Among such candidate genes could be several tRNA genes or the sup45-400 allele, as introduction of wild-type SUP45 to Isp ${ }^{+}$strains has been shown to mimic the Isp ${ }^{-}$phenotype [13]. To the extent of our knowledge, this is the second reported case of nonsense suppressor phenotype modulated by aneuploidy, the first being chromosome VIII disomy elevating translation termination efficiency due to SPB1 duplication [16].

Finally, there is a question why many of the isolates checked in this work contain additional chromosomes. Our data do not allow us to estimate the frequency of chromosome gain, but still the proportion of aneuploid isolates in our analysis, the variability of karyotypes and non-integer coverage data suggest significant genome plasticity. The 25-25-2V-P3982 strain possesses missense mutations in SUP35 and SUP45. These genes have been linked to chromosome stability [32], but as we also found additional chromosome I in a SUP35 SUP45 strain ([psi $\left.\left.{ }^{-}\right]\right)$, we consider sup35-25 or sup45-400 an unlikely cause of the genome instability. In an attempt to find other genes which could be connected to genome instability in 25-25-2V-P3982, we compared the list of 46

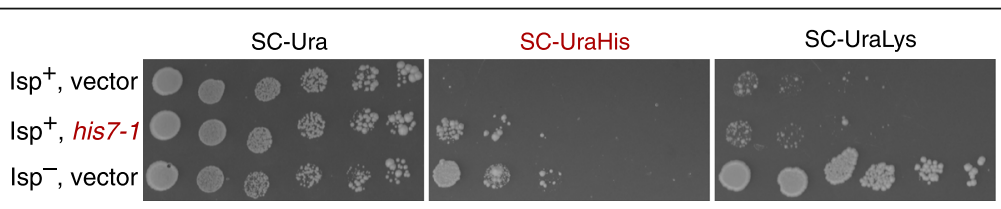

Fig. 5 An additional copy of his7-1 improves growth of an ssp $^{+}$isolate (p3) on histidine dropout medium. Shown are five-fold serial dilutions for representative clones starting from equal number of cells, after 14 days of incubation. Vector, pRS316. his7-1, pRS316-his7-1 
genes with premature stop codons found in the genome of this strain [33] to the curated list of 692 chromosome instability genes [32]. Intersection of these lists returned three genes, $A D E 1, M N L 1$ and $C N N 1$. The ade1-14 allele has been used as a marker of nonsense suppression in multiple works [26] without reports on genome instability, and in addition, it is partially suppressed in the strain used; thus, we consider ade1-14 an unlikely contributor to the observed chromosome instability. MNL1, encoding a protein residing in the endoplasmic reticulum and acting in glycoprotein degradation [34], was detected to influence genome stability in A-like faker screen [35], but the mechanism behind this effect is unclear. The third gene, CNN1, encodes a kinetochore component; deletion of this gene had been associated with chromosome segregation defects in several works $[35,36]$. CNN1 contains a premature stop codon in 25-25-2V-P3982 but not in other related strains of the Peterhof genetic collection [33] and might contribute to the observed chromosome instability.

\section{Conclusions}

1. In our experimental system, amplification of chromosome II confers nonsense suppressor phenotype and guanidine hydrochloride resistance at the cost of overall decreased viability in rich medium.

2. SFP1 might represent a novel regulator of chromosome stability, as SFP1 overexpression elevates frequency of the additional chromosome loss in our system.

3. Prolonged treatment with guanidine hydrochloride leads to selection of resistant isolates, some of which are disomic for chromosome II.

\section{Methods}

\section{Strains and cultivation}

P-2V-P3982 (clone $\left[P S I^{+}\right]$) (MAT $\alpha$ ade1-14 his7-1 lys287 ura3 $\triangle 0$ thr4-B15 leu2-B2 $\left[P^{+} I^{+}\right]$) was derived from 2V-P3982 [10] with transient SUP35 overexpression (Kirill V. Volkov, unpublished data). Clone 25 ascends to the 25-2V-P3982 strain (MAT $\alpha$ ade1-14 his7-1 lys2-87 ura3 $\Delta 0$ thr4-B15 leu2-B2 sup35-25 sup45-400) described earlier [10]. 2-P-2V-P3982 (clone [psi $\left.{ }^{-}\right]$) is derived from P-2V-P3982 with passaging on YEPD medium with $5 \mathrm{mM}$ guanidine hydrochloride three times. $25-25-2 \mathrm{~V}$ P3982 (MATa ade1-14 his7-1 lys2-87 ura340 thr4-B15 leu2-B2 sup35-25 sup45-400) was described earlier [11, 13, 33]; generation of isolates is summarized in Fig. 2. Either PSL2 [37] or p1 genomic DNA was used as euploid control in aCGH experiments.

Standard yeast media $[38,39]$ with modifications were used. Guanidine hydrochloride (Sigma G-3272-100G) was added to a final concentration of $5 \mathrm{mM}$. Yeast strains were cultivated at $26^{\circ} \mathrm{C}, \mathrm{E}$. coli cells were cultivated at $37^{\circ} \mathrm{C}$.

Yeast transformation was carried out according to the standard protocol [40] with modifications.

\section{Plasmids}

Cloning was carried out according to standard protocols [41, 42]. To obtain pRS316-his7-1, his7-1 with flanking regions was PCR amplified from genomic DNA of $\mathrm{p} 3$ with primers HIS7_F_HindIII_(GTAACAAGCTTTCTTTCCT CTACCACTGCCAA) and HIS7_R_HindIII_ (ACCATAA GCTTTGGTACAATTTCTCCAAGCTG) and ligated into RS316 [43] at HindIII sites. In addition to a nonsense mutation A299T leading to premature stop codon at position 77 [44], it contains a PCR-induced substitution T947C leading to a missense mutation L316P. pRS426-SFP1, a pRS426 [45] derivative containing SFP1 under the control of its own promoter, was described earlier [11]. pRS316 [43] and pRS426 [45] were also used as control vectors.

\section{DNA extraction and analysis}

DNA extraction for whole genome sequencing, library preparation and data analysis methods was described earlier [33]. DNA extraction for PCR was performed as described in [38]. DNA extraction for aCGH, labeling and hybridization were performed as described in [46] except that yeast strains were grown at $22{ }^{\circ} \mathrm{C}$ for four days. Custom 8x15k design (AMID 028943) Aligent arrays were used; the slides were scanned and then analyzed with GenePix Pro 6.0 software. In each case, phenotype of the clones used for nucleic acid extraction was checked at the moment of extraction with spotting on selective media.

\section{Data analysis and availability}

Microarray data analysis was performed in R [47] with limma [48]; ggplot2 [49] was used for plotting. Bowtie2 [50] was used for short read alignment. Mapping coverage was plotted with Qualimap [51]. aCGH data were analysed with CGH-Miner Excel add-in [52]. Details of next generation sequencing data analysis and expression microarray data analysis are provided in $[24,33]$, respectively.

\section{Additional files}

Additional file 1: Figure S1. Expression values for all the genes disomic strains relative to wild-type control from [25] compared to expression values of the $\mathrm{Isp}^{-}$isolate $(\mathrm{m} 2)$ relative to an $\mathrm{Isp}^{+}$one $(\mathrm{p} 2)$ sorted by chromosome. Analysis as in Fig. 1. (PNG $611 \mathrm{~kb}$ )

Additional file 2: Table S1. Expression values for all genes in an Ispisolate ( $\mathrm{m} 2$ ) relative to an $\mathrm{Isp}^{+}$one (p2) and mean coverage in approximately $1 \mathrm{~kb}$-long windows for an $\mathrm{Isp}^{-}$isolate $(\mathrm{m} 2)$ relative to an $\mathrm{Isp}^{+}$one (p3). Genes/ windows are sorted by chromosomal position. Genes or windows located on duplicated chromosomes are in bold. (XLS $1215 \mathrm{~kb}$ )

Additional file 3: Figure S2. Graphical summary of aCGH results for each isolate tested. (a) Schematic represenation of the results combined 
with the information on relationship of the strains (see Fig. 2). aCGH, microarray-based comparative genomic hybridization. WGS, whole genome sequencing. (b) Summarized data for each isolate. Horizontal lines represent chromosomes; red signifies amplified regions while green signifies deleted regions. Clones or strains used as experimental and control samples are indicated on the top of each page. (PDF $3621 \mathrm{~kb}$ )

Additional file 4: Table S2. Raw aCGH data for each isolate tested. (XLS $3589 \mathrm{~kb})$

\section{Abbreviations}

aCGH: Microarray-based comparative genomic hybridization; GuHCl: Guanidine hydrochloride; PCR: Polymerase chain reaction

\section{Acknowledgements}

The authors would like to express their gratitude to Mikhail Belousov and Polina Lipaeva for critical reading of the manuscript and useful suggestions and other lab members for discussions. We are extremely grateful to Thomas Petes for reagents and the possibility to carry out CGH experiments, to Margaret Dominska and Anthony Moore for technical help, and to Petes lab members for discussions. The research was supported by research resource center "Molecular and cell technologies" of St. Petersburg State University.

\section{Declarations}

This article has been published as part of BMC Genetics Vol 17 Suppl 3, 2016: Selected articles from BGRSISB-2016: genetics. The full contents of the supplement are available online at https://bmcgenet.biomedcentral.com/ articles/supplements/volume-17-supplement-3.

\section{Funding}

The authors acknowledge the Saint-Petersburg State University for grants 15.61.2218.2013, 1.37.291.2015 and 1.42.1394.2015 and the Presidential Grant Council for the grant NSh-9513.2016.4 for funding this work. The publication cost is covered by the authors.

\section{Availability of data and materials}

The datasets supporting the conclusions of this article are available in the NCBI SRA repository, SRR2533174 (https://www.ncbi.nlm.nih.gov/sra/ SRR2533174/) for isolate p3, and SRR2533175 (https://www.ncbi.nlm.nih.gov/ sra/SRR2533175/) for isolate m2, and in the NCBI GEO repository, GSE52189 (http://www.ncbi.nlm.nih.gov/geo/query/acc.cgi?acc=GSE52189) and are included within the article and its Additional files 1, 2, 3 and 4. Genome browser for combined data for the 25-25-2V-P3982 genome is available as a custom hub at the UCSC genome browser (http://genome.ucsc.edu/cgi-bin/ hgHubConnect\#publicHubs; connect Peterhof_yeasts).

\section{Authors' contributions}

PD and LM initiated the project. PD performed the experiments. PD, LM and $\mathrm{GZ}$ analyzed the data and wrote the manuscript. All authors read and approved the final manuscript.

\section{Competing interests}

The authors declare that they have no competing interests.

\section{Consent for publication}

Not applicable.

\section{Ethics approval and consent to participate}

Not applicable.

\section{Author details}

'Department of Genetics and Biotechnology, St. Petersburg State University, 199034, Universitetskaya nab. 7-9, St. Petersburg, Russia. ${ }^{2}$ Laboratory of Amyloid Biology, St. Petersburg State University, 199034, Universitetskaya nab. 7-9, St. Petersburg, Russia.

\section{Published: 22 December 2016}

\section{References}

1. Warner J. Synthesis of ribosomes in Saccharomyces cerevisiae. Microbiol Rev. 1989:53:256-71. Available from: http://mmbr.asm.org/content/53/2/256.short.
2. Woolford JL, Baserga SJ. Ribosome biogenesis in the yeast Saccharomyces cerevisiae. Genetics. 2013;195:643-81. Available from: http://www.genetics. org/content/195/3/643.short.

3. Valente $L$, Kinzy TG. Yeast as a sensor of factors affecting the accuracy of protein synthesis. Cell Mol Life Sci. 2003;60:2115-30. Available from: http://link.springer.com/article/10.1007\%2Fs00018-003-2334-2.

4. Inge-Vechtomov S, Zhouravleva G, Philippe M. Eukaryotic release factors (eRFs) history. Biol Cell. 2003;95:195-209. Available from: http://doi.wiley. com/10.1016/S0248-4900\%2803\%2900035-2.

5. Tuite MF, Cox BS. The genetic control of the formation and propagation of the $\left[\mathrm{PSI}^{+}\right]$prion of yeast. Prion. 2007;1:101-9. Available from: http://www. tandfonline.com/doi/abs/10.4161/pri.1.2.4665.

6. Volkov KV, II'mov EA, Mironova LN, Inge-Vechtomov SG. [The characteristics of the prion-like element of yeast - mutant factor [PSI]. Dokl Akad Nauk. 1997; 357:123-5. Available from: http://www.ncbi.nlm.nih.gov/pubmed/9471272.

7. Moskalenko S, Chabelskaya S, Inge-Vechtomov S, Philippe M, Zhouravleva G. Viable nonsense mutants for the essential gene SUP45 of Saccharomyces cerevisiae. BMC Mol Biol. 2003;4:1-15. Available from: http://www.biomedcentral. com/1471-2199/4/2

8. Moskalenko SE, Zhuravleva GA, Soom MI, Shabel'skaia SV, Volkov KV, Zemlianko OM, et al. Characterization of missense mutations in the SUP45 gene of Saccharomyces cerevisiae encoding translation termination factor eRF1. Genetika. 2004;40:599-606. Available from: http://link.springer.com/ article/10.1023/B:RUGE.0000029148.58151.91.

9. Chabelskaya S, Kiktev D, Inge-Vechtomov S, Philippe M, Zhouravleva G. Nonsense mutations in the essential gene SUP35 of Saccharomyces cerevisiae are non-lethal. Mol Genet Genomics. 2004;272:297-307. Available from: http://link.springer.com/article/10.1007\%2Fs00438-004-1053-1.

10. Volkov KV, Aksenova AY, Soom MJ, Osipov KV, Svitin AV, Kurischko C, et al. Novel non-Mendelian determinant involved in the control of translation accuracy in Saccharomyces cerevisiae. Genetics. 2002;160:25-36. Available from: http://www.genetics.org/content/160/1/25.

11. Rogoza T, Goginashvili A, Rodionova S, Ivanov M, Viktorovskaya O, Rubel A, et al. Non-Mendelian determinant $\left[/ S P^{+}\right]$in yeast is a nuclear-residing prion form of the global transcriptional regulator Sfp1. Proc Natl Acad Sci U S A. 2010;107: 10573-7. Available from: http://www.pnas.org/content/107/23/10573.

12. Romanova NV, Chernoff YO. Hsp104 and prion propagation. Protein Pept Lett. 2009;16:598-605. Available from: http://www.ncbi.n/m.nih.gov/pmc/ articles/PMC2791106/.

13. Aksenova AY, Volkov KV, Rovinsky NS, Svitin AV, Mironova LN. Phenotypic expression of epigenetic determinant $\left[/ \mathrm{SP}^{+}\right]$in Saccharomyces cerevisiae depends on the combination of sup35 and sup45 mutations. Mol Biol. 2006;40:758-63. Available from: http://link.springer.com/article/10.1134\%2FS0026893306050104.

14. Cox BS. PSI, a cytoplasmic suppressor of super-suppressor in yeast. Heredity. 1965;20:505-21. Available from: http://www.nature.com/hdy/journal/v20/n4/ abs/hdy196565a.html.

15. Wickner RB. [URE3] as an altered URE2 protein: evidence for a prion analog in Saccharomyces cerevisiae. Science. 1994;264:566-9. Available from: http://science.sciencemag.org/content/264/5158/566.long.

16. Zadorsky SP, Sopova YV, Andreichuk DY, Startsev VA, Medvedeva VP, IngeVechtomov SG. Chromosome VIII disomy influences the nonsense suppression efficiency and transition metal tolerance of the yeast Saccharomyces cerevisiae. Yeast. 2015:32:479-97. Available from: http://onlinelibrary.wiley.com/doi/10.1002/yea.3074/abstract.

17. Yona AH, Manor YS, Herbst RH, Romano GH, Mitchell A, Kupiec M, et al. Chromosomal duplication is a transient evolutionary solution to stress. Proc Natl Acad Sci U S A. 2012;109:21010-5. Available from: http://www.pnas.org/ content/109/51/21010.long.

18. Hose J, Yong CM, Sardi M, Wang Z, Newton MA, Gasch AP. Dosage compensation can buffer copy-number variation in wild yeast. Elife. 2015;4 e05462. Available from: http://elifesciences.org/content/4/e05462v3.

19. Strope P, Skelly D. The 100-genomes strains, an S. cerevisiae resource that illuminates its natural phenotypic and genotypic variation and emergence as an opportunistic pathogen. Genome Res. 2015;25:1-13. Available from: http://genome.cshlp.org/content/early/2015/04/03/gr.185538.114.abstract.

20. Bergström A, Simpson JT, Salinas F, Barré B, Parts L, Zia A, et al. A highdefinition view of functional genetic variation from natural yeast genomes. Mol Biol Evol. 2014;31:872-88. Available from: http://mbe.oxfordjournals.org/ content/31/4/872.full.

21. Deregowska A, Skoneczny M, Adamczyk J, Kwiatkowska A, Rawska E, Skoneczna $\mathrm{A}$, et al. Genome-wide array-CGH analysis reveals YRF1 gene copy number 
variation that modulates genetic stability in distillery yeasts. Oncotarget. 2015;6: 30650-63. Available from:dx.doi.org/10.18632/oncotarget.5594.

22. Alberti S, Halfmann R, King O, Kapila A, Lindquist S. A systematic survey identifies prions and illuminates sequence features of prionogenic proteins. Cell Elsevier. 2009;137:146-58. Available from: http://www.cell.com/fulltext/ S0092-8674(09)00266-9.

23. Kelly AC, Busby B, Wickner RB. Effect of domestication on the spread of the $\left[\mathrm{PIN}^{+}\right]$prion in Saccharomyces cerevisiae. Genetics. 2014;197:1007-24. Available from: http://www.genetics.org/content/197/3/1007.long.

24. Drozdova P, Rogoza T, Radchenko E, Lipaeva P, Mironova L. Transcriptional response to the $\left[/ \mathrm{SP}^{+}\right]$prion of Saccharomyces cerevisiae differs from that induced by the deletion of its structural gene, SFP1. FEMS Yeast Res. 2014;14:1160-70. Available from: http://femsyr.oxfordjournals.org/content/14/8/1160.long.

25. Dephoure N, Hwang S, O'Sullivan C, Dodgson SE, Gygi SP, Amon A, et al. Quantitative proteomic analysis reveals posttranslational responses to aneuploidy in yeast. Elife. 2014;3:e03023. Available from: https://elifesciences. org/content/3/e03023.

26. Liebman SW, Chernoff YO. Prions in yeast. Genetics. 2012;191:1041-72. Available from: http://www.genetics.org/content/191/4/1041.long.

27. $\mathrm{Xu} Z$, Norris D. The SFP1 gene product of Saccharomyces cerevisiae regulates G2/M transitions during the mitotic cell cycle and DNA-damage response. Genetics. 1998;150:1419-28. Available from: http://www.genetics.org/ content/150/4/1419.

28. Aksenova A, Muñoz I, Volkov K, Ariño J, Mironova L. The HAL3-PPZ1 dependent regulation of nonsense suppression efficiency in yeast and its influence on manifestation of the yeast prion-like determinant [ISP $\left.{ }^{+}\right]$. Genes Cells. 2007;12:435-45. Available from: http://onlinelibrary.wiley.com/doi/10. 1111/j.1365-2443.2007.01064.x/abstract.

29. Sirr A, Cromie GA, Jeffery EW, Gilbert TL, Ludlow CL, Scott AC, et al. Allelic variation, aneuploidy, and nongenetic mechanisms suppress a monogenic trait in yeast. Genetics. 2015;199:247-62. Available from: http://www. genetics.org/content/199/1/247.

30. Gong H, Romanova NV, Allen KD, Chandramowlishwaran P, Gokhale K, Newnam GP, et al. Polyglutamine toxicity is controlled by prion composition and gene dosage in yeast. PLoS Genet. 2012;8:e1002634. Available from: http://journals.plos.org/plosgenetics/article?id=10.1371/ journal.pgen.1002634.

31. Libuda DE, Winston F. Amplification of histone genes by circular chromosome formation in Saccharomyces cerevisiae. Nature. 2006:443:1003-7. Nature Publishing Group. Available from: http://dx.doi.org/10.1038/nature05205.

32. Stirling PC, Bloom MS, Solanki-Patil T, Smith S, Sipahimalani P, Li Z, et al. The complete spectrum of yeast chromosome instability genes identifies candidate CIN cancer genes and functional roles for ASTRA complex components. PLoS Genet. 2011;7:e1002057. Available from: http://journals. plos.org/plosgenetics/article?id=10.1371/journal.pgen.1002057.

33. Drozdova PB, Tarasov OV, Matveenko AG, Radchenko EA, Sopova JV, Polev DE, et al. Genome sequencing and comparative analysis of Saccharomyces cerevisiae strains of the Peterhof genetic collection. PLoS One. 2016;11: e0154722. Available from: http://journals.plos.org/plosone/article?id=10.1371/ journal.pone.0154722.

34. Nakatsukasa K, Nishikawa S, Hosokawa N, Nagata K, Endo T. Mnl1p, an alpha -mannosidase-like protein in yeast Saccharomyces cerevisiae, is required for endoplasmic reticulum-associated degradation of glycoproteins. J Biol Chem. 2001;276:8635-8. Available from: http://www.jbc.org/content/276/12/8635.

35. Yuen KWY, Warren CD, Chen O, Kwok T, Hieter P, Spencer FA. Systematic genome instability screens in yeast and their potential relevance to cancer. Proc Natl Acad Sci U S A. 2007;104:3925-30. Available from: http://www. pnas.org/content/104/10/3925.full.

36. Measday V, Baetz K, Guzzo J, Yuen K, Kwok T, Sheikh B, et al. Systematic yeast synthetic lethal and synthetic dosage lethal screens identify genes required for chromosome segregation. Proc Natl Acad Sci U S A. 2005;102: 13956-61. Available from: http://www.pnas.org/content/102/39/13956.

37. Lee PS, Greenwell PW, Dominska M, Gawel M, Hamilton M, Petes TD. A finestructure map of spontaneous mitotic crossovers in the yeast Saccharomyces cerevisiae. PLoS Genet. 2009;5:e1000410. Available from: http://journals.plos.org/ plosgenetics/article?id=10.1371/journal.pgen.1000410.

38. Kaiser C, Michaelis S, Mitchell A. Methods in Yeast Genetics. NY: Cold Spring Harbor Laboratory Press; 1994.

39. Zakharov I, Kozhin S, Kozhina T, Fedorova I. Methods in yeast Saccharomyces cerevisiae genetics. Leningrad: Nauka; 1984
40. Gietz RD, Schiestl RH. High-efficiency yeast transformation using the LiAc/SS carrier DNA/PEG method. Nat Protoc. 2007;2:31-4. Available from: http:// www.nature.com/nprot/journal/v2/n1/full/nprot.2007.13.html.

41. Maniatis T, Fritsch EF, Sambrook J, et al. Molecular cloning: a laboratory manual. NY: Cold Spring Harbor Laboratory Cold Spring Harbor; 1989.

42. Inoue $\mathrm{H}$, Nojima $\mathrm{H}$, Okayama $\mathrm{H}$. High efficiency transformation of Escherichia coli with plasmids. Gene. 1990;96:23-8. Available from: http:// www.sciencedirect.com/science/article/pii/037811199090336P.

43. Sikorski RS, Hieter P. A system of shuttle vectors and yeast host strains designed for efficient manipulation of DNA in Saccharomyces cerevisiae. Genetics. 1989;122:19-27. Available from: http://www.genetics.org/content/ 122/1/19.abstract.

44. Chabelskaya S, Gryzina V, Moskalenko S, Le Goff C, Zhouravleva G. Inactivation of NMD increases viability of sup 45 nonsense mutants in Saccharomyces cerevisiae. BMC Mol Biol. 2007;8:71. Available from: http://www.biomedcentral.com/1471-2199/8/71.

45. Christianson TW, Sikorski RS, Dante M, Shero JH, Hieter P. Multifunctional yeast high-copy-number shuttle vectors. Gene. 1992;110:119-22. Available from: http://www.sciencedirect.com/science/article/pii/037811199290454W.

46. Zhang H, Zeidler AFB, Song W, Puccia CM, Malc E, Greenwell PW, et al. Gene copy-number variation in haploid and diploid strains of the yeast Saccharomyces cerevisiae. Genetics. 2013;193:785-801. Available from: http://www.genetics.org/content/193/3/785.long.

47. R Core Team. R: A Language and Environment for Statistical Computing. Vienna, Austria; 2015. Available from: http://www.r-project.org/. Accessed 10 Dec 2015.

48. Smyth GK. Limma: linear models for microarray data. In: Gentleman R, Carey V, Dudoit S, Irizarry R, Huber W, editors. Bioinforma. Comput. Biol. Solut. Using $\{R\}$ Bioconductor. New York: Springer-Verlag; 2005. p. 397-420. Available from: http://www.statsci.org/smyth/pubs/limma-biocbook-reprint.pdf

49. Wickham H. ggplot: Elegant Graphics for Data Analysis. New York: SpringerVerlag; 2009.

50. Langmead B, Salzberg SL. Fast gapped-read alignment with Bowtie 2. Nat Methods. 2012;9:357-9. Available from: http://www.nature.com/nmeth/ journal/v9/n4/full/nmeth.1923.html.

51. Okonechnikov K, Conesa A, García-Alcalde F. Qualimap 2: advanced multisample quality control for high-throughput sequencing data. Bioinformatics. 2015;32:btv566. Available from: http://bioinformatics.oxfordjournals.org/ content/32/2/292.long

52. Wang P, Kim Y, Pollack J, Narasimhan B, Tibshirani R. A method for calling gains and losses in array CGH data. Biostatistics. 2005;6:45-58. Available from: http://biostatistics.oxfordjournals.org/content/6/1/45.abstract.

\section{Submit your next manuscript to BioMed Central and we will help you at every step:}

- We accept pre-submission inquiries

- Our selector tool helps you to find the most relevant journal

- We provide round the clock customer support

- Convenient online submission

- Thorough peer review

- Inclusion in PubMed and all major indexing services

- Maximum visibility for your research

Submit your manuscript at www.biomedcentral.com/submit
Biomed Central 